\title{
Biotrin assay to monitor cervical cancer exposure rates
}

A small Irish biotech company has licensed technology from the US National Cancer Institute (NCI) (Box 1), with the aim of carving out a niche within one of the most notable vaccine introductions in recent years. Dublin-based Biotrin has gained access to a series of immunoassays that can be used to guarantee the integrity of the cervical cancer immunization programs using either Gardasil, which recently gained FDA and EMEA approval, or Cervarix, approval of which is presumed to be imminent.

Both are recombinant vaccines against multiple subtypes of human papilloma virus (HPV).

Gardasil's post-launch uptake appears rapid. It racked up $\$ 70$ million in sales in the third quarter of 2006 and was added to the US Centers for Disease Control and Prevention's Vaccines for Children program on November 1 . Both vaccines are expected to attain blockbuster status, but the commercial aims for Biotrin's assays will be smaller by several orders of magnitude. "Big for us is ten or twenty million dollars," says Biotrin CEO Cormac Kilty. Nonetheless, use of the assays could be central to efforts to track the effectiveness of immunization programs.

According to the Geneva-based World Health Organization (WHO), HPV infection is linked to over $99 \%$ of cervical cancer cases. Around $100 \mathrm{HPV}$ genotypes have been identified, of which $40 \%$ can infect the male or female genital area. Gardasil, developed by Whitehouse Station, New Jersey-based Merck, and Cervarix, developed by London-based GlaxoSmithKline, offer protection against the HPV16 and HPV18 subtypes, which are responsible for around $70 \%$ of all cervical cancer cases. (Gardasil also protects against HPV6 and HPV11, which cause more than $90 \%$ of outbreaks of genital warts.)

Although screening programs to detect the cervical cancers caused by other HPV genotypes will still need to continue in parallel with immunization efforts, use of the two vaccines should eventually lead to a drop of roughly $70 \%$ of the incidence of cervical cancer, after accounting for the regional variation in the prevalence of differing HPV genotypes, in those countries with universal coverage.

As with all vaccine introductions, however, there is still work to be done in understanding how the vaccines perform in the field and in ensuring that batches of vaccine are handled appropriately in the distribution chain. John Schiller, principal investigator at the Laboratory of Cellular Oncology at the $\mathrm{NCI}$, whose research forms the basis of the technologies licensed by Merck, GSK and now Biotrin, says after four to five years' experience with human immunizations, the vaccines appear to offer a solid defense. "There's reason to hope protection may be long term," he says. "We're precisely at that point where we just have to wait and see."

Common international biological, proficiency and quality standards that would support ongoing global monitoring of HPV infection - of all types - and of HPV immunization programs are still in the process of being finalized, although this effort should be completed this year. "It's necessary to have data from various countries being reported in

\section{Box 1 Navigating the NIH}

Biotrin's agreement with the $\mathrm{NCl}$ came about with the help of a technology transfer executive from an Irish government agency that supports indigenous companies, who undertook a year-long placement at the National Institutes of Health campus. Brian O'Neill, senior development adviser at Dublin-based Enterprise Ireland, spent his time in Bethesda screening licensing opportunities for Irish life sciences firms and learning how to navigate the NIH's complex maze of extramural funding programs. "The biggest criticism - for want of a better word-of the NIH is that nobody knows where to go," he says.

assays that are traceable to international standards," says Joakim Dillner, professor of virology and molecular epidemiology at Malmo University Hospital in Malmo, Sweden. "Everything should be traceable to one bottle." Dillner's lab was appointed the first WHO global HPV reference laboratory in September. Regional reference laboratories are also being established in each of the WHO's six geographical regions, which will support efforts being undertaken at the national level, although it will take some time for a global network of labs to be fully up and running, Dillner says. "Availability of a serum assay from a commercial company will make all this standardization work for the network very much easier," he points out.

Biotrin's Kilty agrees. "People would like to have a standardized GMP [good manufacturing practices]-produced test," he says. The company plans to develop four tests in total-two separate enzyme-linked immunosorbent assays for detecting antibodies that bind to HPV16 and HPV18, plus two neutralization assays to detect antibodies that actually neutralize HPV16 and HPV18. They will be available in about a year.

The binding assays, Schiller says, will provide a rapid measure of HPV16 and HPV18 exposure rates within a population and provide healthcare authorities with an impetus for launching immunization campaigns. The neutralization assays are based on the same virus-like particle (VLP) technology developed at the NCI on which the actual HPV vaccines are based. Each VLP incorporates a plasmid that encodes an alkaline phosphatase reporter gene. Neutralizing antibodies present in a vacinee's serum block the pseudovirus's infection of and replication within a standard cell line, which is indicated by the absence of a phosphorylation signal (Virology 321, 205216, 2004).

The high cost of the new vaccines is likely to limit their initial availability in low-income countries, which register around $80 \%$ of the 500,000 new cases reported annually. However, patents covering the vaccine technology have not been taken out in developing countries, so it may be possible to diversify production, Schiller says, and make it available at a lower cost in regions with limited healthcare budgets.

Cormac Sheridan, Dublin 